\title{
Primary Aldosteronism: The Next Five Years
}

\author{
Author \\ John W. Funder \\ Affiliation \\ Hudson Institute of Medical Research and Monash \\ University, Clayton, Australia \\ Key words \\ prevalence, discovery, harmonization, agenda, \\ cottage industry, aldosterone \\ received 06.08 .2017 \\ accepted 07.09.2017 \\ Bibliography \\ DOI https://doi.org/10.1055/s-0043-119802 \\ Published online: 24.10.2017 \\ Horm Metab Res 2017; 49: 977-983 \\ (c) Georg Thieme Verlag KG Stuttgart · New York \\ ISSN 0018-5043 \\ Correspondence \\ John W. Funder \\ 64 Parker Road \\ Wandin East \\ 3139 Victoria \\ Australia \\ Tel.: +61/359/642 341, Fax: +61/359/643 651 \\ John.funder@hudson.org.au
}

\section{Introduction}

Currently the management of primary aldosterone is a cottage industry. For those with an historical bent, before the industrial revolution in the eighteenth century consumer goods were produced by individual farms, ateliers, workshops - cottages, not corporations. In the present day, a dozen grandmothers making apple pies for the church fair is a residual example of a cottage industry - more or less sugar, different sorts of apples, some with cinnamon, others with ginger; all as their mothers and grandmothers taught them. Hallowed by tradition, very difficult to change.

When we look at the current management of primary aldosterone there are well over a dozen possible variations between centers. In order, as follows:

- Assays: for plasma aldosterone concentration (PAC), ELISA or mass spec; PAC versus 24-h urinary aldosterone plus metabolites; plasma renin activity (PRA) or plasma renin concentration (PRC); and in the near future plasma angiotensin.

- Screening: aldosterone to renin ratio (PAC)/(PRA) or (PAC)/ PRC; setting (or not) an arbitrary lower limit for renin, despite

\section{ABSTRACT}

The management of primary aldosteronism is widely varied within various published guidelines, with very little in the way of data supporting the choice of one variation over others. Current estimates of prevalence are probably accurate for aldosterone producing adenoma, but fall very short of that for bilateral adrenal hyperplasia. Discovery at the level of basic science has proven illuminating over the past 6 years in terms of unilateral disease and both somatic and germline mutations, with much less focus on the much more common bilateral disease; Attempts at harmonization have begun - for example, criteria for complete/partial/absent cure after adrenalectomy for unilateral disease; again focus on bilateral disease is muted. A number of possibilities are suggested as agenda for active consideration and change, across a wide range of areas - referral patterns, screening, confirmation and lateralization, what will be needed is discussion and agreement, to fill the lacunae within the current guidelines. Those involved will want to change to make such an agenda possible. the ability to measure lower levels with confidence; use (or not) of an arbitrary value for PAC, usually in the high normal range, below which screening is deemed negative, despite an indicative aldosterone to renin ratio (ARR).

- Cut-offs: setting high limits for ARR and elevated aldosterone (strict criteria), thus lowering the percentage of patients positive on screening and subsequent testing; more relaxed criteria give $\sim$ double the number of patients testing positive.

- Confirmatory testing: standard (recumbent) saline infusion; oral sodium loading; fludrocortisone and salt loading suppression test; captopril challenge; furosemide and upright posture; seated saline suppression test; dexamethasone enhanced fludrocortisone suppression test...

- Imaging: computed tomography (CT), magnetic resonance imaging (MRI), positron emission tomography (PET), ${ }^{11} \mathrm{C}$-metomidate, chemokine receptor type 4 (CRCX-4).

- Adrenal venous sampling: with or without Cosyntropin; if with, Cosyntropin dose, bolus and/or infusion; selective or super selective catheterization; selectivity index, lateralization 
index; utility or otherwise of contralateral suppression; intraprocedural cortisol measurement; cortisol, androstenedione, metanephrine as denominators.

- Medical therapy: spironolactone dose-limited or not/adjuvant amiloride/ other adjuvant antihypertensives.

It is tempting to refer to this as infinite variation, which is not scientifically accurate. There are, however, well over 12 common variations, and factorial twelve is a very large number.

Where do we go from here? What, if anything, can be achieved in the next five years? In an attempt to address these questions, they will be considered under four headings - recognition, discovery, harmonization and finally agenda. Hope springs eternal...

\section{Primary Aldosteronism: Recognizing the Prevalence}

As noted above, currently some centers use strict criteria, others more relaxed. Those using strict criteria commonly report the prevalence of PA to be $5-6 \%$ of hypertensives among whom $\sim 3 \%$ are ultimately shown to have an aldosterone producing adenoma (APA), and 2-3\% bilateral adrenal hyperplasia (BAH). Centers using more relaxed diagnostic criteria report $3-4 \%$ to have an APA, and $6-10 \% \mathrm{BAH}$. This difference is not surprising, given that commonly APA are at the more florid end of primary aldosteronism, and are in themselves illuminating. What they signify is that - perhaps understandably - patients with unilateral disease (APA) are a much more attractive area for management than those with $\mathrm{BAH}$. Efficient lateralisation followed by unilateral laparoscopic adrenalectomy produces very satisfying results: biochemical cure (normalization of PAC/plasma [K+]/ARR) in almost $100 \%$ of patients, complete clinical remission in at least half (particularly in females and young patients) and partial clinical success (normotension with lower drug dosage) in nearly all the remainder. The lure is cure, and patients with $\mathrm{BAH}$ who require lifelong management and maintenance are not nearly as satisfying.

That said, even though APA patients tend to have higher levels of PAC, what evidence there is suggests that there is little difference in terms of cardiovascular risk between the two groups, as recently reported [1]. What is also becoming recognized is that whereas both the strict and relaxed criteria are effective in picking APA, (the 'low-hanging fruit'), in fact neither adequately identifies the extent to which BAH contributes to primary aldosteronism. This is not new, as a study by Helber et al. from almost 40 years ago illustrates [2]. Three groups of patients were sodium loaded for 6 days ( 175 meq orally) at the end of which a 24-h urine collection was made. In the 56 control patients, all urinary aldosterone values were $\leq 6 \mu \mathrm{g}$, which was thus taken as the upper limit of the normal range. All 16 confirmed primary aldosterone patients (interestingly, $12 \mathrm{APA}$ and $4 \mathrm{BAH}$, the latter with lesser but still elevated urinary aldosterone values) had levels well above the normal range. Of 100 'essential' hypertensives, 64 had urinary levels within the normal range - that is, $\leq 6 \mu \mathrm{g} /$ day - and were termed group A; 36 (Group B) showed urinary aldosterone levels $>6 \mu \mathrm{g} /$ day, overlapping with the established $\mathrm{BAH}$ group. Group A patients had an average plasma $\left[\mathrm{K}^{+}\right.$] of $4.26 \pm 0.37$ (SD) meq/l, and Group B patients
$3.81 \pm 0.44(p<0.001)$. Group A patients given spironolactone showed a $9 \mathrm{mmHg}$ fall in blood pressure, in contrast with those with established primary aldosteronism $(21 \mathrm{mmHg})$ and those in Group B $(22 \mathrm{mmHg})$. Taken together, the patients with elevated urinary aldosterone $(52 / 116)$ constituted $45 \%$ of the patients with hypertension.

These were not isolated studies, but followed others with similar findings, a number in German, now almost completely forgotten [3-9]. What brought back the issue of prevalence front and center was the development by Gouli et al. of a dexamethasoneenhanced fludrocortisone suppression test (FDST) for confirmation/exclusion of primary aldosteronism in hypertensive patients [10]. Seventy-two non-hypertensive individuals with normal adrenals on CT underwent an FDST, with the 97.5 percentile thus established taken as the upper limit of normal (serum aldosterone $74 \mathrm{pM} / \mathrm{I}$, aldosterone/renin ratio $32 \mathrm{pM} / \mathrm{l} / \mathrm{mU} / \mathrm{I})$. On this basis 180 hypertensives with $(n=44)$ and without $(n=136)$ adrenal adenomas on CT underwent an FDST: 31 \% of these showed values for both serum aldosterone and aldosterone/renin ratio above the upper limit of normal, as established in the control group.

Current thinking about the drivers of aldosterone secretion focuses largely on the renin-angiotensin system, with plasma $\left[\mathrm{K}^{+}\right]$ often an afterthought, and ACTH - a powerful but not chronic stimulus - nowhere. The results of the FDST can be interpreted to suggest that we ignore ACTH at our peril in the diagnosis of 'autonomous aldosterone secretion', that is, primary aldosteronism. An additional role for ACTH was shown in subsequent studies by Markou et al. from the same group [11], on hypertensive patients whose serum aldosterone/aldosterone to renin ratios were within the normal limits established in the first study. Normal controls ( $n=61$ ) and hypertensives (without PA on FDST; $n=113$ ) were subjected to ultra low-dose $(0.03 \mu \mathrm{g})$ ACTH stimulation and a treadmill test. Of the hypertensives, 30/113 (27\%) showed an aldosterone but not a cortisol hyper-response to ACTH infusion, with no difference in basal ACTH/renin concentrations or cortisol response compared with controls or non-hyper-responders. On the treadmill test, hyper-responders showed higher levels of aldosterone, and higher values for aldosterone/renin and aldosterone/ACTH than nonresponders or controls. If of every 100 hypertensives $31 \%$ have PA on FDST, and $27 \%$ of the $69 \%$ who do not are hyper-responders to ACTH ( $n=19)$, the value for hypertensives with overproduction of aldosterone is $50 \%$, strikingly similar to that found by Helber et al. over three decades previously.

There is also increasing evidence that the prevalence of primary aldosteronism is not confined to patients with hypertension. Early evidence for this came from a case-controlled study by Stowasser et al. on the rare condition of familial hyperaldosteronism Type I. Young normotensive patients with $\mathrm{FH}-\mathrm{I}(\mathrm{n}=8)$ were matched 1:3 for blood pressure with controls and shown to have both structural and functional cardiac damage [12]. Vasan and colleagues showed in patients in the Framingham study that over time blood pressure increased most in patients with the highest levels of serum aldosterone [13]. Reports of demonstrable primary aldosteronism in prehypertensives came initially from Japan [14], with a subsequent European longitudinal study detailing the development of primary aldosteronism over five years in initially normotensive subjects [15]. Most recently in a landmark paper on 
two counts, Baudrand and colleagues (from Boston and Chile) examined 210 normotensives from the HyperPATH cohort who had suppressed plasma renin activity (PRA $<1.0 \mathrm{ng} / \mathrm{ml} / \mathrm{h}$ ), and had completed an oral sodium suppression test, received an infusion of angiotensin II, and had blood pressure and renal plasma flow determined [16]. On the generous criteria for salt-loaded urinary aldosterone excretion rate $(12 \mu \mathrm{g} / 24 \mathrm{~h}$ : cf. Helber et al. [2] $6 \mu \mathrm{g} / 24 \mathrm{~h})$ the frequency of biochemically confirmed primary aldosteronism was $29 / 210$ (14\%).

There are two particularly noteworthy aspects to the study. First is that "Primary aldosteronism (PA) is defined as a severe form of autonomous aldosterone secretion that causes hypertension". This opening sentence of the abstract is tempered by their subsequent description of the $14 \%$ frequency of "normotensives with PA". The authors then write that "our findings indicate a continuum of subclinical, autonomous and renin-independent aldosterone secretion and mineralocorticoid receptor activation that ranges from subtle to biochemically confirmed PA". Quite so: and the inference to be drawn is that the strict definition of primary aldosteronism is no longer tenable. The most interesting finding of the study, however, is that there was no difference in plasma aldosterone/renin ratios between the $14 \%$ of "normotensives with PA" and the remaining $86 \%$.

Finally, in terms of prevalence, there is now increasing acceptance that hypertension resistant to 3 conventional agents including a diuretic (or requiring 4 or more agents for normotension) not only includes the $15-20 \%$ with primary aldosteronism as currently defined, but perhaps most if not all such patients: certainly the response to mineralocorticoid receptor antagonists is equivalent in the $15-20 \%$ and the residual $80-85 \%$. Similarly, there is growing acceptance that many subjects designated 'low renin hypertensives' in fact are victims of very straitened cut-offs: certainly their responses to mineralocorticoid receptor antagonists are identical, in terms of lowering of blood pressure and left ventricular hypertrophy, to those seen with confirmed primary aldosteronism [17].

\section{Primary Aldosteronism: Prognostications for Discovery}

Over a five-year period, there is ample room for progress in what we know. There follows a personal exercise of crystal-ball gazing, with the hope that there will be one or more discoveries that nobody could foresee, and which come as a total surprise.

- There may or may not be yet uncharacterized somatic mutations in APA. The initial estimates in Caucasian APA patients will perhaps be shifted upwards, reflecting more refined sequencing; the very high reported values of KCNJ5 mutations ( $70-80 \%$ of all APA) in Japan and China may reflect a real ethnic difference, or more probably selection/referral bias.

- Of the familial forms of hyperaldosteronism, FH-I, FH-III and $\mathrm{FH}-\mathrm{IV}$ are characterized at the genomic level; to date, despite strenuous attempts, $\mathrm{FH}$-II is not. Over the next five years this is likely to split in two ways - one is the characterization of the genomic basis of FH-II in at least some of the very large kindreds which have been documented, and the other is the realization that as the increased prevalence of primary aldosteronism is acknowledged there is an increasing possibility that first degree relatives might have different underlying causative mechanisms. The fact that one family member has an APA, and another BAH, does not exclude a common cause, but raises the index of suspicion that this might not be the case.

- In five years, it is likely that there will be available validated and accurate assays for plasma angiotensin. An equally confident prediction would be that it will be much longer than 5 years for this proximate actor in aldosterone secretion to be widely adopted.

- Within five years it is likely that there will be at least some novel mineralocorticoid receptor antagonists which are tissue-selective. Whether or not these will replace spironolactone at low dose as the common mineralocorticoid receptor antagonist in primary aldosteronism is not at all certain. Eplerenone is expensive, except in Japan, varyingly recompensable, and optimally prescribed twice daily: unfortunately, from the primary aldosterone viewpoint, many of the mineralocorticoid receptor antagonists in development do not appear natriuretic, a sine qua non for primary aldosteronism. Watch this space, but keep expectations low.

- At the level of basic science/translational research, in five years we should know more about how aldosterone producing cell clusters (APCC) emerge more or less to replace a continuous zona glomerulosa with advancing age, in most but not all subjects [18], and what drives them to produce aldosterone. Although often called constitutive in terms of aldosterone secretion, adrenal glands in the elderly, many of whom appear to have little or no zona glomerulosa, do respond to salt restriction by substantial elevation of plasma aldosterone, albeit to a lesser extent than in younger individuals [18]. In apparently normal adrenal glands some APCC express somatic mutations (e.g., CACNA1D, ATP1A1) but curiously not KCNJ5, by far the most common of all somatic mutations [19].

- In step with the question of what drives APCC is an older question, which is what drives $\mathrm{BAH}$. Germline mutations are one clear but relatively uncommon cause of $\mathrm{BAH}$; for the bulk of bilateral disease, true hyperplasia or bilateral multinodular disease, we do not have as yet convincing answers. There have been preliminary suggestions of roles for activating antibodies against angiotensin receptors, of super-sensitivity to angiotensin, and of leptin. In a series of early studies patients with BAH (and a small subset with APA) were shown to be hyper-responsive to angiotensin infusion, with this hyperresponsiveness not further characterized [20-23]. In many senses, BAH is the poor relation, given the extent of focus on APA: this is not only unwarranted, but counterproductive given the increasing recognition of the high prevalence of non-APA primary aldosteronism, despite attempts to flag this as 'autonomous aldosterone secretion' or 'dysregulated aldosterone production.'

- Finally, and given the emerging evidence that primary aldosteronism causes or contributes to blood pressure elevation in $\geq 50 \%$ of so-called essential hypertensives (classical, plus elevated 24-h urinary aldosterone 
levels/resistant hypertension/low renin hypertension/FDST/ ACTH super sensitivity), methods to obviate adrenal venous sampling (AVS) for as many patients as possible are crucial. Lateralisation by AVS is needed as a guide to unilateral adrenalectomy: currently, lateralisation on the basis of imaging is demonstrably inferior [24]. The problem is that of removing the wrong adrenal, a major blunder: the occasional APA missed, and treated medically, is a source of dissatisfaction, but not a blunder. The current Endocrine Society Guideline supports omitting AVS in a young patient with immeasurably low renin, clearly elevated PAC, and a classic unilateral adenoma on imaging. Much more important is to establish and validate algorithms maximizing the probability of bilateral disease, so that such patients can proceed to medical therapy without AVS. Of all the prognostications for discovery this last is the most urgent, and if successfully developed over the next five years clearly the most useful in terms of management.

\section{Primary Aldosteronism: Harmonization}

As previously noted, there is wide variation in practice: harmonisation is unlikely to be achieved across the board in five years, but some key areas in screening and diagnosis could well be largely aligned within that time frame: where there's a will there's a way. Areas in which such harmonization might be achieved are as follows:

- Screening: the report by Baudrand et al. [16] where $14 \%$ of normotensives had clearly demonstrated primary aldosteronism on the basis of their elevated urinary aldosterone levels but no elevation in aldosterone/renin ratio when a spot plasma aldosterone value is used should sound the tocsin for the present ARR as a screening test. The physiology underling this discrepancy can be seen in $\mathbf{F i g . ~ 1 , ~ s h o w n ~ b e l o w . ~}$

- The salient majority of aldosterone secretion is in recumbency, possibly reflecting redistribution of blood volume and consequent generation of angiotensin, possibly involving factors yet to be established. It differs from cortisol, where the nocturnal rise is both ACTH driven, and constitutes a much lesser percentage of total daily secretion; in contrast, daytime secretion of aldosterone is clearly a small minority of 24-h secretion. The half-life of aldosterone in blood is of the order of $12 \mathrm{~min}$, so that measuring levels three hours into the waking day can be confidently expected to miss nocturnal hypersecretion. What this demands at first blush is a return to the past, to the days of Helber et al. previously cited, and to screen patients with a higher probability of primary aldosteronism (in the 2016 Guidelines [25], 50\% of hypertensives) by a more representative aldosterone concentration. The circadian physiology of aldosterone essentially invalidates 'low-renin hypertension', for example, on the basis of a positive ARR but a spot plasma aldosterone concentration<14.5 ng/dl (in [17], for example).

- Physicians believe that 24-h urine collections are disliked by patients, which may be the case. In the face of this difficulty, it would be a worth a trial of a 24-h collection versus the first morning collection, plus any urine passed on waking during the night. If, as might be expected, a high level of concord-

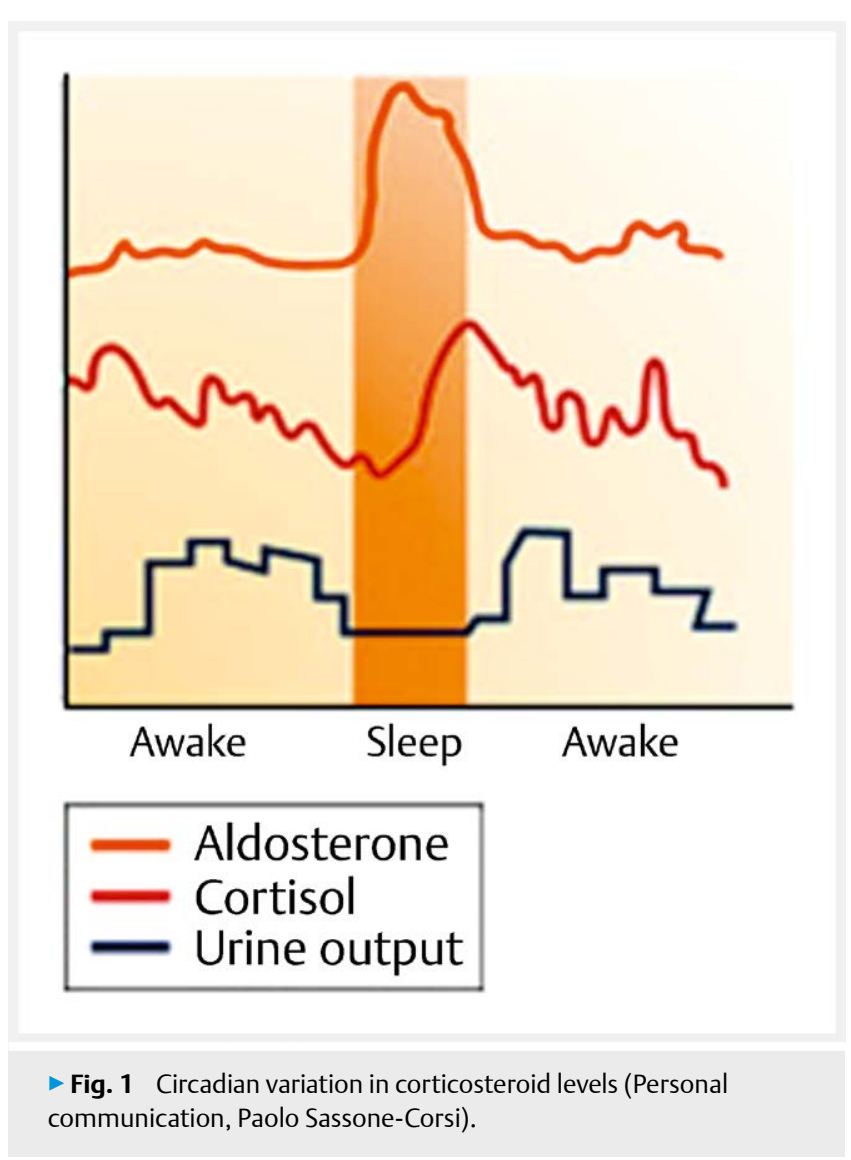

ance was established, the overnight rather than the complete 24-h collection could be used to estimate whether aldosterone production is or is not within the normal range. This is urgent, in that it is probable that the majority of patients with primary aldosteronism currently screen negative because of a totally inappropriate spot plasma aldosterone measurement.

- One of the outstanding examples of variation betweencenters is that of the extraordinary range of methods used to confirm/exclude primary aldosteronism in subjects who screen positive in terms of the aldosterone/renin ratio. The seated saline suppression test (SSST) has proven very promising in preliminary studies [26], on which basis some centers have already chosen to adopt it. If it is validated by larger studies it would then be worthwhile doing a trial of the SSST with and without dexamethasone at $11 \mathrm{PM}$ on the eve of the test, to determine whether the findings on the FDST are replicated.

- The use of cosyntropin in AVS is vexed, and the relative advantages and disadvantages it confers are debated, on occasion hotly. One measure of harmonization might be to standardize the timing, dosage and administration (infusion \pm bolus) among those centers in which it is used.

- Adopt/adapt standard criteria for biochemical success of unilateral adrenalectomy after AVS, and for complete, partial and absent clinical success. In a recent study using the Delphi process and iterative questionnaires consensus was reached [27]. The rationale in particular was to examine whether the previously reported wide variation in clinical success reflected 
different criteria or not. The answer appears to be a resounding no, in that the range of outcomes across 12 expert centers was as broad as that pre-consensus, so that the variation reflects other factors (e. g., age at referral, gender bias, etc.,). Such criteria need to be real-world: one example is the breadth of acceptance of blood pressure measurements allowed (office/home/24h ambulatory).

- Finally, recognize that low dose (maximum 50 mg/day; preferably $12.5-25 \mathrm{mg} /$ day) spironolactone, plus amiloride if available and considered needed, plus other anti-hypertensives as required, is optimal therapy for $\mathrm{BAH}$. Low dose spironolactone thus supported is much less likely to cause unwanted side-effects, and thus much less likely to provoke issues of poor compliance.

\section{Primary Aldosteronism - Agenda}

Many items that need reconsideration and action have already been telegraphed in the previous sections; five such agenda items are thus briefly listed, as follows:

- Recognize the true prevalence of primary aldosteronism to include 'dysregulated aldosterone secretion' and 'inappropriate aldosterone production'.

- Recalibrate the discovery/management/therapeutic focus from the present APA front and center to include large-scale follow-up studies to optimize management of the much more common BAH.

- Given recognition of the increased prevalence of primary aldosteronism, and high probability that BAH constitutes the majority of such patients, to refine and validate procedures and protocols to avoid AVS in such patients.

- Lose the spot plasma aldosterone assay, given the preponderance of aldosterone secretion being overnight, and the probability that aldosterone hypersecretion is similarly predominantly nocturnal.

- If the seated saline suppression test preliminary results are confirmed in a current larger study, to use the SSST in parallel with the existing confirmatory test at each individual center, with a view to all centers using a common test in the future.

To which might be added six additional agenda items not previously raised which may cause less pain and have more chance of success that the five major issues previously addressed. In order:

- Reconsider how we manage partial clinical success after unilateral adrenalectomy for APA. Patients with complete biochemical success after laparoscopic adrenalectomy, in terms of normalizing plasma $\mathrm{PAC} /\left[\mathrm{K}^{+}\right] / \mathrm{ARR}$ often still require antihypertensives to reduce blood pressure into the normal range; this is termed partial clinical success. Continued elevation of blood pressure despite complete biochemical success is commonly attributed to vascular damage/coexistent essential hypertension. A modest minority of such patients show low plasma aldosterone concentrations post-operatively, which commonly revert to normal over weeks or months; these low levels presumably reflect suppression of aldosterone biosynthesis in the contralateral adrenal, and the time taken to recover. When, however, plasma aldosterone concentrations are normal post-surgery for APA, there are powerful reasons for including a low-dose mineralocorticoid receptor antagonist in the antihypertensive regimen. One of the difficulties entailed in referring to MR as 'aldosterone receptors' is that were that the case, and the source of aldosterone hypersecretion removed, there would appear to be little sense in using an 'aldosterone blocker' (sic). In fact MR evolved millions of years before aldosterone [28]; 90-99\% of all MR are normally occupied but not activated by cortisol, which has the same affinity for MR as aldosterone but circulates at 100-fold higher plasma free concentrations; and finally, crucially, in conditions of tissue damage cortisol becomes an MR agonist, mimicking aldosterone [29]. The vasculature is a physiologic target for aldosterone; in damaged blood vessels cortisol mimics aldosterone and raises blood pressure [30]. Finally, in DOC/salt rats, eplerenone has been shown to reverse established vascular and cardiac damage to control levels [31]. There would thus appear to be a strong case for including a low-dose mineralocorticoid receptor antagonist in the antihypertensive regime for patients with partial clinical success after unilateral adrenalectomy for primary aldosteronism when their plasma aldosterone levels are in the normal range.

- Reconsider how we manage BAH. In several remote communities with very low salt intake plasma aldosterone concentrations are correspondingly very high, with no deleterious cardiovascular effects. Elevated aldosterone concentrations per se are thus not the problem, when they are homeostatic: in conjunction with inappropriate sodium status, however, the combination is vasculotoxic and blood pressure elevating. Accordingly, part of the regimen of treatment of BAH should logically include tapering off of salt intake over 6 weeks, to a maintainable low-salt regimen: (it takes 6 weeks for the taste buds to recalibrate).

- Educate general practitioners/family doctors about the prevalence of primary aldosteronism (even on the current straitened definition). In a recent study of Italian and German General Practitioners [32], respondents reported having hundreds of hypertensive patients, as might be anticipated, on average with remarkably high average office blood pressure. In Germany $18 \%$ of GPs reported having no patients with primary aldosteronism, and in Italy the figure was $30 \%$. Referrals were overwhelmingly to cardiologists, rather than to endocrinologists or expert hypertension centers; in Germany, the figure for prevalence was $2 \%$, and in Italy $1 \%$.

- Close the gap between the diagnosis of hypertension and referral for possible primary aldosteronism. This is commonly 5-10 years, and there are compelling data that clinical success complete or partial - is closely negatively correlated with advancing age [27]. The laminated two-page single sheet desk reference/patient reference in development for the Endocrine Society recommends that at the first visit to the GP at which an elevated blood pressure is found a careful blood draw for plasma renin is made. If renin activity is $<1.0 \mathrm{ng} / \mathrm{ml} / \mathrm{h}$ 
or renin concentration $<15 \mathrm{mU} / \mathrm{l}$, the patient should be immediately referred to either a dedicated hypertension center or a specialist endocrinologist, to be screened for primary aldosteronism.

- Throw off the shackles of patriarchy. There are no data showing a higher prevalence of primary aldosteronism in males than females - and good indications for a female preponderance in APAs harboring the very common (35-50\%) KCNJ5 somatic mutation [33, 34]. There are, however, a number of centers in which the referral pattern is overwhelmingly (70-80\%) male. This is not the fault of the centers to which such referrals are made, but the cultural assumptions of the referring physicians. These assumptions result in double jeopardy, in that at all ages women with an APA removed by unilateral adrenalectomy are much more likely to have complete or partial (together $>95 \%$ ) clinical success than agematched men [28]. What many of the present referral practices do is not just inequitable on a gender basis, but in addition denies the chance of successful intervention to the group considerably more likely to profit from it.

- Finally, the last of this second group of agenda items flows from a political statement made over five centuries ago:

"There is nothing more difficult and dangerous, or more doubtful of success, than to attempt to introduce a new order of things... for the innovator has for enemies all those who derive advantage from the old order, while those who will benefit from the new will be at best lukewarm defenders" [35].

Machiavelli's rather bleak view of human nature notwithstanding, the final agenda item is to continue to bring together those involved in primary aldosteronism, so that we can increasingly reach consensus, for the benefit of the patients involved and the societies in which we live.

\section{Conflict of Interest}

The author declares no conflict of interest.

\section{References}

[1] Monticone S, Burrello ], Tizzani D, Bertello C, Viola A, Buffolo F, Gabetti L, Mengozzi G, Williams TA, Rabbia F, Veglio F, Mulatero P. Prevalence and clinical manifestations of primary aldosteronism encountered in primary care practice. J Am Coll Cardiol 2017; 69: $1811-1820$

[2] Helber A, Wambach G, Hummerich W, Bönner G, Meurer KA, Kaufmann W. Evidence for a subgroup of essential hypertensives with non-suppressible excretion of aldosterone during sodium loading. Klin Wochenschr 1980; 58: 439-447

[3] Collins RD, Weinberger MH, Dowdy A], Nokes GW, Gonzales CM, Luetscher JA. Abnormally sustained aldosterone secretion during salt loading in patients with various forms of benign hypertension, relation to plasma renin activity. J Clin Invest 1970; 49: 1415

[4] Grim CE, Peters THJ. Low renin hypertension: A state of inappropriate secretion of aldosterone. J Lab Clin Med 1971; 78: 816
[5] Fournier A, Lagrue G, Baulon A, Nakache JP, Kazandjian M, Legrand JC, Menard J. Significance and prediction of spironolactone effectiveness in essential hypertension. In: Extrarenal activity of aldosterone and its antagonists. Excerpta Medica; Amsterdam 1972; 54

[6] Leutscher JA, Beckerhoff R, Dowdy A], Wilkinson R. Incomplete suppression of aldosterone secretion and plasma concentration $n$ hypertensive patients on high sodium intake. In Genest Koiw W. (eds) Hypertension 72. Berlin: Springer; 1972: 286

[7] Klaus D, Klumpp F, Zehner J. Primary hypertension with low plasma renin level. Dtsch Med Wochenschr 1973; 42: 1980-1987

[8] Helber A, Meurer KA, Rosskamp E, Wambach G, Dickmans HA, Kaufmann W. Aldosterone excretion and plasma renin activity in patients with essential hypertension and with primary aldosteronism. Klin Wochenschr 1974; 52: 966-973

[9] Kloppenburg PWC, Drayer JIM, Benraad HB, Benraad Th]. Normal aldosterone versus supranormal aldosterone hypertension: And alternative to normal renin versus low renin hypertension. In Distler W. (ed) Current Problems in Hypertension. Stuttgart: Thieme; 1974: 143

[10] Gouli A, Kaltsas G, Tzonou A, Markou A, Androulakis II, Ragkou D, Vamvakidis K, Zografos G, Kontogeorgos G, Chrousos GP, Piaditis G. High prevalence of autonomous aldosterone secretion among patients with essential hypertension. Eur J Clin Invest 2011; 41: 1227-1236

[11] Markou A, Sertedaki A, Kaltsas G, Androulakis II, Marakaki C, Pappa T, Gouli A, Papanastasiou L, Fountoulakis S, Zacharoulis A, Karavidas A, Ragkou D, Charmandari E, Chrousos GP, Piaditis GP. Stress-induced aldosterone hyper-secretion in a substantial subset of patients with essential hypertension. J Clin Endocrinol Metab 2015; 100: 2857-2864

[12] Stowasser M, Sharman J, Leano R, Gordon RD, Ward G, Cowley D, Marwick TH. Evidence for abnormal left ventricular structure and function in normotensive individuals with familial hyperaldosteronism type I. J Clin Endocrinol Metab 2005; 90: 5070-5076

[13] Vasan RS, Evans JC, Larson MG, Wilson PW, Meigs JB, Rifai N, Benjamin EJ, Levy D. Serum aldosterone and the incidence of hypertension in nonhypertensive persons. N Engl J Med 2004; 351: 33-41

[14] Ito Y, Takeda R, Karashima S, Yamamoto Y, Yoneda T, Takeda Y. Prevalence of primary aldosteronism among prehypertensive and stage 1 hypertensive subjects. Hypertens Res 2011; 34: 98-102

[15] Markou A, Pappa T, Kaltsas G, Gouli A, Mitsakis K, Tsounas P, Prevoli A, Tsiavos V, Papanastasiou L, Zografos G, Chrousos GP, Piaditis GP. Evidence of primary aldosteronism in a predominantly female cohort of normotensive individuals: A very high odds ratio for progression into arterial hypertension. J Clin Endocrinol Metab 2013; 98: 1409-1416

[16] Baudrand R, Guarda F], Fardella C, Hundemer G, Brown J, Williams G, Vaidya A. Continuum of renin-independent aldosteronism in normotension. Hypertension 2017; 69: 950-956

[17] Ori Y, Chagnac A, Korzets A, Zingerman B, Herman-Edelstein M, Bergman M, Gafter U, Salman H. Regression of left ventricular hypertrophy in patients with primary aldosteronism/low-renin hypertension on low-dose spironolactone. Nephrol Dial Transplant 2013; $1-5$

[18] Nanba K, Vaidya A, Williams GH, Zheng I, Else T, Rainey WE. Age-related autonomous aldosteronism. Circulation 2017; 136: 347-355

[19] Nishimoto K, Tomlins SA, Kuick R, Cani AK, Giordano TJ, Hovelson DH, Liu C], Sanjanwala AR, Edwards MA, Gomez-Sanchez CE, Nanba K, Rainey WE. Aldosterone-stimulating somatic gene mutations are common in normal adrenal glands. Proc Natl Acad Sci USA 2015; 112 : E4591-E4599

[20] Wisgerhof M, Brown RD. Increased adrenal sensitivity to angiotensin II in low renin essential hypertension. J Clin Invest 1978; 61: 1456-1462

[21] Carey RM, Ayers CR, Vaughan ED Jr., Peach M], Herf SM. Activity of [des-aspartyl1]-angiotensin II in primary aldosteronism. J Clin Invest 1979; 69: 718-726 
[22] Wisgerhof M, Brown RD, Hogan M], Carpenter PC, Edis A]. The plasma aldosterone response to angiotensin II infusion in aldosterone-producing adenoma and idiopathic hyperaldosteronism. J Clin Endocrinol Metab 1981; 52: 195-198

[23] Gordon RD, Gomez-Sanchez CE, Hamlet SM, Tunny T], Klemm SA. Angiotensin-responsive aldosterone-producing adenoma masquerades as idiopathic hyperaldosteronism (IHA: adrenal hyperplasia) or low-renin essential hypertension. J Hypertens Suppl 1987; 5: S103-S106

[24] Rossi GP, Funder JW. Adrenal Venous sampling versus computed tomographic scan to determine treatment in primary aldosteronism (The SPARTACUS Trial): A critique. Hypertension 2017; 3: 396-397

[25] Funder JW, Carey RM, Mantero F, Murad MH, Reincke M, Shibata H, Young WF Jr. The management of primary aldosteronism: case detection diagnosis and treatment: An endocrine society clinical practice guideline. J Clin Endocrinol Metab 2016; 101: 1889-1916

[26] Ahmed AH, Cowley D, Wolley M, Gordon RD, Xu S, Taylor PJ, Stowasser $M$. Seated saline suppression testing for the diagnosis of primary aldosteronism: A preliminary study. J Clin Endocrinol Metab 2014; 99: 2745-2753

[27] Williams TA, Lenders ], Mulatero P, Burrello ], Rottenkolber M, Adolf C, Fumitoshi S, Amar L, Quinkler M, Deinum J, Beuschlein F, Kitamoto KK, Pham U, Morimoto R, Umakoshi H, Prejbisz A, Kocjan T, Naruse M, Stowasser M, Nishikawa T, Young WF Jr., Gomez-Sanchez CE, Funder JW, Reincke M. Outcomes after adrenalectomy for unilateral primary aldosteronism: An International consensus on outcome measures and analysis of remission rates in an international cohort. Lancet Diabetes Endocrinol 2017; 5: 689-699

[28] Kassahn KS, Ragan MA, Funder JW. Mineralocorticoid receptors: Evolutionary and pathophysiological considerations. Endocrinology 2011; 152: 1883-1890
[29] Mihailidou AS, Loan Le TY, Mardini M, Funder JW. Glucocorticoids activate cardiac mineralocorticoid receptors during experimental myocardial infarction. Hypertension 2009; 54: 1306-1312

[30] Levy DG, Rocha R, Funder JW. Distinguishing the antihypertensive and electrolyte effects of eplerenone. J Clin Endocrinol Metab 2004; 89: 2736-2740

[31] Young M, Funder JW. Eplerenone, but not steroid withdrawal, reverses cardiac fibrosis in deoxycorticosterone/salt-treated rats. Endocrinology 2004; 145: 3153-3157

[32] Mulatero P, Monticone S, Burrello ], Veglio F, Williams TA, Funder ]. Guidelines for primary aldosteronism: uptake by primary care physicians in Europe. J Hypertens 2016; 34: 2253-2257

[33] Choi M, Scholl UI, Yue P, Björkland P, Zhao B, Nelson-Williams C, Ji W, Cho Y, Patel A, Men C], Lolis E, Wisgerhof MV, Geller DS, Mane S, Hellman P, Westin G, Åkerström G, Wang W, Carling T, Lifton RP. K+ channel mutations in adrenal aldosterone-producing adenomas and hereditary hypertension. Science 2011; 331: 768-772

[34] Boulkroun S, Beuschlein F, Rossi GP, Golib-Dzib JF, Fischer E, Amar L, Mulatero P, Samson-Couterie B, Hahner S, Quinkler M, Fallo F, Letizia C, Allolio B, Ceolotto G, Cicala MV, Lang K, Lefebvre H, Lenzini L, Maniero C, Monticone S, Perrocheau M, Pilon C, Plouin PF, Rayes N, Seccia TM, Veglio F, Williams TA, Zinnamosca L, Mantero F, Benecke A, Jeunemaitre X, Reincke M, Zennaro MC. Prevalence, clinical, and molecular correlates of KCNJ5 mutations in primary aldosteronism. Hypertension 2012; 59: 592-598

[35] Machiavelli The Prince. 1513 